\title{
THE QUANTUM BOLTZMANN EQUATION IN A NONTRIVIAL BACKGROUND
}

\author{
M. JOYCE \\ LPT, Université Paris-XI, Bâtiment 211, F-91405 Orsay Cedex, France \\ E-mail: Michael.Joyce@th.u-psud.fr \\ K. KAINULAINEN \\ NORDITA, Blegdamsvej 17, DK-2100, Copenhagen Ø, Denmark \\ E-mail: kainulai@nordita.dk \\ T. PROKOPEC \\ Université de Lausanne, Institut de physique théorique, BSP, CH-1015 Lausanne, \\ Suisse \\ E-mail: Tomislav.Prokopec@ipt.unil.ch
}

\begin{abstract}
This talk $a$ is a status report on our study of quantum transport equations relevant for baryogenesis computations. Our main finding is that, as a consequence of localization in space, the quasiparticle picture of the plasma dynamics breaks down at first non-trivial order in gradient expansion. While in this talk we focus on bosons, we expect that a similar picture holds for fermions. We then argue that the quasiparticle picture is recovered in the adiabatic limit of frequent scattering.
\end{abstract}

${ }^{a}$ Talk delivered by T. Prokopec at COSMO-99, Trieste, Italy, Sep 27 - Oct 2, 1999; Report No. UNIL-IPT/00-02 (Feb 2000)

\section{Introduction and motivation}

A reliable computation of baryon production at the electroweak phase transition requires a new formulation of the dynamics of the $\mathrm{CP}$ violating sources in an out-of-equilibrium relativistic plasma. To this purpose we are working on controlled derivation of the relativistic quantum transport equations for weakly coupled plasmat. 20. These equations are essential to a systematic treatment of the matter-antimatter creation at the electroweak phase transition, since they describe the relevant $\mathrm{CP}$ violating sources, their transport and dissipation in one formalism. Our aim is to apply this formalism to computation of the baryon production at the electroweak transition in the Standard Model and its supersymmetric extensions, and thus make contact with the upcoming particle physics experiments, most notably LHC, DAPHNE, PEP-II, etc.

An essential ingredient in our method is an expansion in gradients of a slowly varying background. It is necessary to go beyond the leading order in gradients, since the $\mathrm{CP}$ violating sources required by any baryogenesis mechanism influence particle dynamics only beyond the leading order. The model 


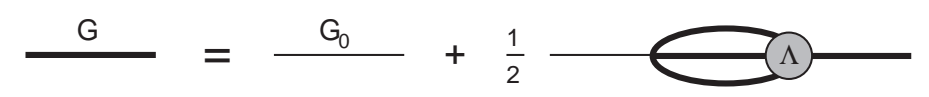

Figure 1: Diagrammatic representation of the Schwinger-Dyson equation for the scalar theory with $\mathcal{L}_{\text {int }}=-\lambda\left(\phi^{\dagger} \phi\right)^{2} / 4$. $G$ and $G_{0}$ denote the full and free propagators, respectively, and $\Lambda$ the four point function.

whose dynamics we study here is very simple, a complex scalar field with the lagrangian

$$
\mathcal{L}=\partial_{\mu} \phi^{\dagger} \partial^{\mu} \phi-\frac{1}{2} m^{2} \phi^{\dagger} \phi+\text { h.c. }+\mathcal{L}_{\text {int }},
$$

where for definiteness we shall take the quartic interaction $\mathcal{L}_{\text {int }}=-\lambda\left(\phi^{\dagger} \phi\right)^{2} / 4$. The mass $m=m\left(x^{\mu}\right)$ represents coupling to a classical background field which varies in space and time, an important example being the Higgs field condensate. Such a background implies breakdown of the translational invariance, which has as a consequence localization in position space, or equivalently, delocalization in momentum space and breakdown of the quasiparticle picture in the plasma. This breakdown is quite different from one related to the collective self-energy corrections. Indeed, assuming a planar symmetry, $m=m(z)$, which models the bubble wall at the electroweak phase transition, we find that at the $p$ th order in gradients the momentum splits into $p+2$ branches ( $c f$. figure 2), while the energy remains conserved. A self-consistent description of the problem requires $p+2$ distinct distribution functions, or equivalently one distribution function and $p+1$ functions that measure coherent quantum densities on phase space, whose dynamics is described by generalized transport equations. In the adiabatic limit (of frequent scattering) the coherent densities are suppressed, and one thus recovers the quasiparticle picture with one semiclassical transport equation for a distribution function which describes the dynamics on a modified shell on phase space.

\section{The Kadanoff-Baym equations}

The Kadanoff-Baym equations (KBE) describe the out-of-equilibrium dynamics of quantum fields, and can be derived from the Schwinger-Dyson equations (SDE) in the Keldysh closed time contour (CTC) formalism 6 . In figure 1 we show the SDE for the scalar theory (Bi). In the weakly coupled limit the four point function can be approximated by $\Lambda \propto \lambda$, so that the SDE for the propagator closes. The self-energy $\Sigma$ is in this case computed in the Born approximation, which is illustrated by the two loop diagram in figure 1 .

Since we are primarily interested in the dynamics of the propagator in the presence of a slowly varying background, we write the KBEs in the mixed 
(Wigner) representation as follows

$$
\begin{array}{cl} 
& \cos \diamond\left\{\Omega^{2} \pm i \omega \Gamma\right\}\left\{G^{r, a}\right\}=1 \\
- & \sin \diamond\left(\left\{\Omega^{2}\right\}\left\{i G^{<,>}\right\}-\left\{i \Sigma^{<,>}\right\}\left\{G_{R}\right\}\right) \\
= & \frac{1}{2} \cos \diamond\left(\left\{\Sigma^{>}\right\}\left\{G^{<}\right\}-\left\{\Sigma^{<}\right\}\left\{G^{>}\right\}\right)
\end{array}
$$

where the operator $\diamond\{f\}\{g\} \equiv \frac{1}{2}\left[\partial_{X} f \cdot \partial_{k} g-\partial_{k} f \cdot \partial_{X} g\right]$ is a generalized Poisson bracket. The PE (2) is the dynamical equation for the retarded and advanced propagators, $G^{r, a}$, while the QBE (3) is the transport equation for the quantum Wigner functions, which in the coordinate representation read

$$
G^{>}(x, y)=-i\left\langle\phi(x) \phi^{\dagger}(y)\right\rangle, \quad G^{<}(x, y)=-i\left\langle\phi^{\dagger}(y) \phi(x)\right\rangle .
$$

Various quantities in equations (2) and (3) are defined as follows

$$
\begin{aligned}
\Omega^{2} & =G_{0}^{-1}-\Sigma_{R} \equiv k^{2}-m^{2}-\Sigma_{R} \\
G^{r, a} & =G_{R} \mp i \mathcal{A}, \quad \Sigma^{r, a}=\Sigma_{R} \mp i \omega \Gamma,
\end{aligned}
$$

where $\Sigma_{R}$ and $\Gamma$ denote the real part of the self-energy and the damping rate, respectively, $\mathcal{A}$ denotes the spectral function defined by

$$
\mathcal{A} \equiv \frac{i}{2}\left(G^{r}-G^{a}\right)=\frac{i}{2}\left(G^{>}-G^{<}\right),
$$

while $G_{R}$ is the real part of the propagator, which is related to the spectral function by the following spectral integral $G_{R}=(1 / \pi) \mathcal{P} \int d \omega / \mathcal{A}\left(\omega^{\prime}\right) /\left(\omega-\omega^{\prime}\right)$. A similar spectral relation holds for $\Sigma_{R}$ and $\omega \Gamma=i\left(\Sigma^{>}-\Sigma^{<}\right) / 2$. Finally, the weak coupling expansion ensures that the self-energies $\Sigma^{<,>}$may be computed in the Born approximation.

The mixed representation is defined by the Wigner transform, which is the Fourier transform with respect to the relative coordinate $r=x-y$. For example, for the propagator we have

$$
G(k ; X)=\int d^{4} r e^{i k \cdot r} G(X+r / 2, X-r / 2),
$$

where $X$ is the average coordinate defined by $X=(x+y) / 2$.

Equations (2) and (3) represent an accurate description of the plasma dynamics background is slowly varying, i.e. when the gradient approximation applies. Formally, this is the case when $\|\diamond\| \ll 1$. Physically, this condition holds when the de Broglie wave length of excitations is small in comparison to 
the characteristic length scale of the background variation. For the electroweak phase boundary the characteristic scale is the bubble wall thickness $L \sim(10-$ 20)/T 6 , whereas for plasma excitations $\partial_{k} \sim k^{-1}$, so that $\|\diamond\| \sim\left\|\partial_{X} \partial_{k}\right\| \sim$ $1 / k L$, implying that the gradient approximation is accurate when $k \gg L^{-1}$, which is close to thermal equilibrium satisfied by most of plasma excitations.

\section{Propagator equation}

We now consider the propagator equation (2). For simplicity we assume a planar symmetry such that $m=m(x)$, and set $\Sigma_{R}=0$. For the electroweak phase transition this case corresponds to a planar bubble wall in the wall frame. To second order in gradients the propagator is solved by 2

$$
G^{r, a} \rightarrow G_{p=2}=\frac{1}{k_{0}^{2}-k_{x}^{2}}+\frac{1}{2} \frac{\left(m^{2}\right)^{\prime \prime}}{\left(k_{0}^{2}-k_{x}^{2}\right)^{3}}-\frac{1}{2} \frac{2 k_{0}^{2}\left(m^{2}\right)^{\prime \prime}+\left[\left(m^{2}\right)^{\prime}\right]^{2}}{\left(k_{0}^{2}-k_{x}^{2}\right)^{4}},
$$

where $k_{0}^{2}=\omega^{2}-k_{\|}^{2}-m^{2}(x)$ denotes the unshifted pole, $\left(m^{2}\right)^{\prime \prime} \equiv \partial_{x}^{2} m^{2}$, and $\left(m^{2}\right)^{\prime} \equiv \partial_{x} m^{2}$. Note that at second order in gradients there is no pole shift, but instead multiple poles emerge. Now let us make the following decomposition of the quantum Wigner functions

$$
G^{<}=-2 i \mathcal{A} n, \quad G^{>}=-2 i \mathcal{A}(n+1),
$$

where $n$ is the generalized distribution function. Recall that the spectral function $\mathcal{A}(6)$ defines the spectrum (poles) of the propagator. For example, at leading order in gradients the spectral function becomes a sharp on-shell projector: $\mathcal{A} \rightarrow \mathcal{A}_{0}=\left(\pi / 2 k_{0}\right)\left[\delta\left(k_{x}-k_{0}\right)+\delta\left(k_{x}+k_{0}\right)\right]$. To understand how is this projective behaviour modified by the presence of the derivative corrections in Eq. (8), we study the following spectral integral

$$
\mathcal{I}_{p}[\mathcal{T}]=\frac{2}{\pi} \int_{-\infty}^{\infty} d k_{x} \mathcal{A}_{p} \mathcal{T} \rightarrow \sum_{i=0}^{p+1} c_{i} \mathcal{T}^{(i)}\left(k_{0}\right) .
$$

Note that the series terminates at the $p+1$ st order in derivatives, where $p$ is the highest order gradient in the propagator $G_{p}$. For example, when $p=2$,

$$
\mathcal{I}_{2}[n]=\frac{n\left(k_{\mathrm{sc}}\right)}{k_{\mathrm{sc}}}+c_{2}\left(\partial_{k_{x}}^{2} n\right)\left(k_{0}\right)+c_{3}\left(\partial_{k_{x}}^{3} n\right)\left(k_{0}\right),
$$

where $k_{x}=k_{\mathrm{sc}}$ defines the semiclassical dispersion relation

$$
k_{\mathrm{sc}}=k_{0}+\frac{1}{8} \frac{\left(m^{2}\right)^{\prime \prime}}{k_{0}^{3}}+\frac{5}{32} \frac{\left[\left(m^{2}\right)^{\prime}\right]^{2}}{k_{0}^{5}} .
$$


This agrees with the dispersion relation obtained by the standard WKB method 2. The additional higher order derivative terms in Eq. (11) signify the breakdown of the quasiparticle picture and emergence of quantum correlations, and they are direct consequence of the multipole structure of $G_{p}$ (8).

\section{Transport equations}

We now discuss the implications of the quasiparticle picture breakdown on transport. To this end we simplify the QBE (3) by keeping only the higher order derivative term contributing to the flow term 3 to obtain

$$
-\left(\diamond-\diamond^{3} / 3\right)\left\{\Omega^{2}\right\}\left\{i G^{<}\right\}=\frac{1}{2}\left(\left\{\Sigma^{>}\right\}\left\{G^{<}\right\}-\left\{\Sigma^{<}\right\}\left\{G^{>}\right\}\right)+o\left(\diamond^{2}, \Gamma\right) .
$$

In order to make use of the decomposition (9) and the properties of the spectral function $\mathcal{A}$, we shall perform spectral integrals weighted by powers of the momentum over Eq. (13). Upon defining the moment functions

$$
f_{l} \equiv \frac{2}{\pi} \int_{-\infty}^{\infty} d k_{x} k_{x}^{l} \mathcal{A}_{p=2} n, \quad(l=0,1,2, . .),
$$

these integrals result in the following set of coupled transport equations

$$
\omega \partial_{t} f_{l}+\tilde{k}_{l} \partial_{x} f_{l+1}-\frac{l}{2}\left(\partial_{x} k_{0}^{2}\right) f_{l-1}-\frac{l(l-1)(l-2)}{48}\left(\partial_{x}^{3} k_{0}^{2}\right) f_{l-3}=\mathrm{Coll}_{l},
$$

where $\mathrm{Coll}_{l}$ is the collision term, which approximately has the following form

$$
\mathrm{Coll}_{l}=-\Gamma^{>} f_{l}+\Gamma^{<}\left(f_{l}+k_{0}^{2-l} \kappa_{l}\right) .
$$

Here $\Gamma^{<,>}$denote the damping rates computed in the Born approximation 3 and, for example, $\kappa_{0}=k_{\mathrm{sc}}$. To any finite order $p$ in the gradient expansion, there are only $p+2$ independent moments and in particular at second order in gradients one can derive the constraint: $f_{4}-4 k_{0} f_{3}+6 k_{0}^{2} f_{2}-4 k_{0}^{3} f_{1}+k_{0}^{5} f_{0}=0$, so that Eqs. (15) close for the first four moments, $l=0,1,2,3$.

The following picture has thus emerged. Due to the higher order poles in the propagator (8), the quasiparticle dispersion relation has split into four branches defined by

$$
k_{x} \equiv \tilde{k}_{l}=k_{0}+\frac{(l-1)(l-2)}{16} \frac{\left(m^{2}\right)^{\prime \prime}}{k_{0}^{3}}+\frac{l^{2}-5 l+5}{32} \frac{\left[\left(m^{2}\right)^{\prime}\right]^{2}}{k_{0}^{5}},
$$

such that for example $\tilde{k}_{0}=k_{\mathrm{sc}}$ in Eq. (12). This is illustrated in figure 2. Note that the splitting diverges in the infrared, where gradient approximation breaks down. For a particle with an energy $\omega$ incoming onto the bubble wall, the momentum splits into four branches, and the full description of the dynamics then requires defining one distribution function on each branch. 


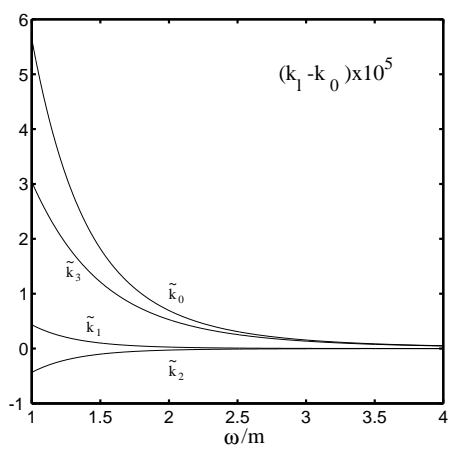

Figure 2: The hypersurfaces $k_{x}=\tilde{k}_{l}$ on which the moments $f_{l}$ flow according to Eqs. (15. 16.).

\section{Quantum coherence and semiclassical limit}

We shall now briefly describe an alternative formulation of the transport equations (15-16), which is convenient for discussion of the semiclassical limit. Let us first define

$$
\begin{aligned}
f & =k_{\mathrm{sc}} f_{0}, & f_{\mathrm{qc} 1}=f_{1}-f \\
f_{\mathrm{qc} 2} & =f_{2} / \kappa_{2}-f_{\mathrm{qc} 1}-f, & f_{\mathrm{qc} 3}=f_{3} / k_{0}^{2}-f_{\mathrm{qc} 2}-f_{\mathrm{qc} 1}-f,
\end{aligned}
$$

which then implies the following transport equation for $f$ :

$$
\omega \partial_{t} f+k_{\mathrm{sc}} \partial_{x}\left(f+f_{\mathrm{qc} 1}\right)=-\Gamma^{>} f-\Gamma^{<}(f+1) .
$$

The distribution function $f$ measures population density of particles on the hypersurface $k_{x}=\tilde{k}_{0} \equiv k_{\mathrm{sc}}$, while the densities $f_{\mathrm{qci}}$ measure the coherent quantum correlations between neighboring hypersurfaces $k_{x}=\tilde{k}_{i-1}$ and $k_{x}=\tilde{k}_{i}$ defined in Eq. (17). Eq. (19) is already in the form of the standard Boltzmann equation, apart from the presence of an unknown function $f_{\mathrm{qc} 1}$. Equations for $f_{0}$ and $f_{1}$ then imply

$$
\omega\left(\partial_{t}+\Gamma_{\mathrm{qc}}\right) f_{\mathrm{qc} 1}+\kappa_{2}-k_{\mathrm{sc}} \partial_{x} f_{\mathrm{qc} 1}+\partial_{x} \kappa_{2}\left(f_{\mathrm{qc} 1}+f_{\mathrm{qc} 2}\right)+\kappa_{2} \partial_{x} f_{\mathrm{qc} 2}=s_{1},
$$

where the source

$$
s_{1}=\left(k_{\mathrm{sc}}-\kappa_{2}\right) \partial_{x} f+\left(\partial_{x} k_{0}^{2} / 2 k_{\mathrm{sc}}\right)-\partial_{x} \kappa_{2} f
$$

represents coherent mixing of $f$ and $f_{\mathrm{qc} 1}$. One can obtain similar equations for the coherent quantum densities $f_{\mathrm{qc} 2}$ and $f_{\mathrm{qc} 3}$. The coherent densities $f_{\mathrm{qci}}$ 
are all damped at the rate $\Gamma_{\mathrm{qc}} \equiv\left(\Gamma^{>}-\Gamma^{<}\right) / \omega$, which is an out-of equilibrium generalization of the on-shell damping rate 6 . Finally, in the frequent scattering limit defined as

$$
\Gamma_{\mathrm{qc}} L \gg 1
$$

one can neglect $f_{\text {cq1 }}$ in Eq. (19) and one obtains a semiclassical fquation in which quasiparticles flow along modified semiclassical trajectories 3 .

\section{Conclusions and outlook}

We have presented a self-consistent derivation of transport equations for scalar theory beyond the leading order in gradients. We found that as a consequence of localization in space, the quasiparticle picture of transport breaks down. We have shown how to self-consistently reformulate transport theory by including information about the varying background to a finite order in gradients. Our result are the transport equations (15) 16), which at second order in gradients describe the dynamics on four momentum branches in phase space. We have also briefly mentioned that one recovers the semiclassical picture in the (adiabatic) limit of frequent scattering.

The question which we are addressing now is how to generalize our results to mixing scalar fields and massive chiral fermions, which are the cases relevant for baryogenesis studies.

\section{References}

1. M. Joyce and K. Kainulainen, and T. Prokopec, in proceedings of SEWM 98, Copenhagen, Denmark, 2-5 Dec 1998, hep-ph/9906413.

2. M. Joyce and K. Kainulainen, and T. Prokopec, to be published in Phys. Lett. B, hep-ph/9910535.

3. M. Joyce and K. Kainulainen, and T. Prokopec, Phys. Lett. B468 (1999) 128, hep-ph/9906411.

4. L.P. Kadanoff and G. Baym, Quantum Statistical Mechanics, Benjamin Press, New York (1962).

5. P. Danielewicz, Ann. Phys. 152 (239) 1984; J. Rammer and H.J. Smith Rev. Mod. Phys. 58 (1986) 323; S. Mrowczynski and U. Heinz, Ann. Phys. 229 (1994) 1; P. Henning, Phys. Rep. 253 (1995) 235.

6. G. Moore and T. Prokopec, Phys. Rev. D52 (1995) 7182.

7. H.A. Weldon, Phys. Rev. D28 (2007) 1983. 